\title{
AMINOFUNCTIONALIZED MESOPOROUSSILICA NANOPARTICLESLOADED WITH 5-FLUOROURACIL
}

\author{
Jijo Thomas*., Kala D and Archana George
}

College of Pharmaceutical Sciences, Govt. Medical College, Thiruvananthapuram

\begin{tabular}{|c|c|}
\hline A R T I C L E I N F O & A B S T R A C T \\
\hline Article History: & We report the synthesis of Amino funtionalized and Mesoporous Silica Nanoparticles \\
\hline Received $19^{\text {th }}$ December, 2016 & and loading of 5-fluorouracil. Themesosporous silica nanoparticles (MSNs) were \\
\hline Received in revised form $24^{\text {th }}$ January, 2017 & $\begin{array}{l}\text { synthesized viasolgel process using cetyltrimethyl ammonium bromide (CIAB) surfactant } \\
\text { template. The prepared nanoparticles were amino functionalized by }\end{array}$ \\
\hline Accepted $12^{\text {th }}$ February, 2017 & 3-Aminopropyltriethoxysilane (APTES) toimprove drug entrapment efficiency. The drug \\
\hline Published online $28^{\text {th }}$ March, 2017 & $\begin{array}{l}\text { loading of 5- fluorouracil was done by backfilling approach. The prepared amino } \\
\text { functionalized APT-MSNs were characterized by Fourier transform Infrared (FTIR) }\end{array}$ \\
\hline Key words: & spectroscopy. Scanning electron microscopy (SEM) and Transmission electron microscopy \\
\hline Mesoporous silica nanoparticles, Amino & ology \\
\hline
\end{tabular}

functionalization, SEM, TEM

Copyright $(2017$ Jijo Thomas., Kala D and Archana George. This is an open access article distributed under the Creative Commons Attribution License, which permits unrestricted use, distribution, and reproduction in any medium, provided the original work is properly cited.

\section{INTRODUCTION}

MSNs are solid materials, which contain hundredsof empty channels (mesopores) arranged in a 2D network of honeycomb-like porous structure. Due to their unique adjustable properties such ashigh surface area $(>700 \mathrm{~m} 2 / \mathrm{g})$, pore volume $(>0.9 \mathrm{~cm} 3 / \mathrm{g})$, and tuneable pore diameter $(2-10$ nm) MSNs can serve asversatiledrug delivery carriers. They present a stable and rigid framework with excellent chemical, thermal, and mechanical stability. Furthermore, both the exterior particleand interior pore surfaces can also be easily functionalized or site-specific delivery.

According to IUPAC classification, mesoporousmaterials will have a pore diameter ranging from 2 to $50 \mathrm{~nm}$. Conventional MSN can load a dose of therapeuticdrug with 200-300 mg (maximally about $600 \mathrm{mg}$ ) drug/1 g silica [1]

MSN synthesisis done mainly by two methods

Sol-gel method: Four ingredients are necessary to formmesoporoussilica materials: a surfactant, silica source, an acid or base catalyst and a solvent like ethanol or water. This process requires two-step consideration: Hydrolysis and condensation. Hydrolysis produced colloidal particles in aqueous solution, which can be stimulated at alkaline and acidic $\mathrm{pH}$. High concentration of amphipilic surfactant assembles into a spherical micelle in water and hydrophilic

*Corresponding author: Jijo Thomas

College of Pharmaceutical Sciences, Govt. Medical College,

Thiruvananthapuram soluble precursor like polysilicic acid or silica acid. By electrostatic and hydrogen bonding interaction, the silica precursor is concentrated at the hydrophilic interface and form an amorphous silica, which is a mold of the mesoporousproduct. Removal of remaining surfactant can be done by calcination and extraction method [2].

Spray drying method [3]

The capacity to encapsulate drugs in nanoparticles can be improved by functionalization. The surface functionalizationis generally needed to load proper type of drug molecules (hydrophobic/hydrophilic or positive/negative charged).

In this work we have synthesized mesosporous silica nanoparticles bysolgel process using CTAB surfactant template. The prepared nanoparticles were amino functionalised for enhanced 5-FU loading using APTES.

\section{LITERATURE REVIEW}

1. Kummel AC et al. has done a review on the synthesis and surface functionalization of silica nanoparticles for nanomedicine.

2. Wang $\mathrm{S}$ et al. developed mesoporoussilica nanoparticles (MSNs) loaded with a poorly watersoluble drugtelmisartan, intended to be orally administered to improve the dissolution rate and enhance the drug loading capacity.

3. Xia T et al. modified the surface of MSN particles by a functional group that enhances cellular uptake and allows nucleic acid delivery in addition to traditional drug delivery. 
4. Lee $\mathrm{C}$ et al. developed intracellular $\mathrm{pH}$-responsive mesoporous silica nanoparticles for the controlled release of anticancer chemotherapeutics.

5. Tummala $\mathrm{S}$ et al. formulated and characterized 5Fluorouracil enteric coated nanoparticles for sustained and localized release in treating colorectal cancer.

\section{MATERIALS}

3-Aminopropyltriethoxysilane were purchased from Tokyo chemical industry Co. Ltd, Toshima, Japan. 5-Fluoruracil and Ninhydrin were purchased from Central drug house(P)Ltd., New Delhi, India.Dialysis Membrane (MWCO 12-14 kDa) were purchased from HiMedia Laboratories Pvt. Ltd., Mumbai, India.

\section{METHOD AND CHARACTERIZATION}

MSN were synthesized using the method of Cai et al [4]. $1 \mathrm{~g}$ of CTAB was dissolved in a mixture of $3.5 \mathrm{ml}$ of $2 \mathrm{M}$ Sodium hydroxide and $480 \mathrm{ml}$ ultrapure water. The mixture was heated to $65^{\circ} \mathrm{C}$ and stirred at $1000 \mathrm{rpm}$ while maintaining the temperature. To the homogenous liquid added $5 \mathrm{ml}$ of TEOS. Stirring was continued for a period of $2 \mathrm{hrs}$. The white slurry produced is separated by centrifugation at $5500 \mathrm{rpm}$. Slurry was washed 3 times with water and dried at $75^{\circ} \mathrm{C}$. The dried slurry was then calcined at $600^{\circ} \mathrm{C}$ for $4 \mathrm{hrs}$. The white powder produced was suspended in $50 \mathrm{ml}$ of water with the aid of ultrasonication. Resuspended nanoparticles were freeze dried to get the free falling MSN.

Zeta potential was determined by the electrophoretic mobility of MSN in U- type tube at $25^{\circ} \mathrm{C}$, using Zetasizer Nano ZS (Malvern Instruments Ltd, Worcestershire, United Kingdom) after suitable dilution with water.

Morphology of MSN was characterized using scanning electron microscope JEOL model JSM - $6390 \mathrm{LV}$ at $20 \mathrm{kV}$

Particle size and structure of optimized MSN were studied using Transmission electron microscopy (TEM). The MSN was uniformly dispersed in ultrapure water, drop casted formvar coated copper grid and allowed to dry over night at room temperature. The grid was then imaged at $80 \mathrm{kV}$ using transmission electron microscope (Hitachi, H-7650)

Nitrogen adsorption-desorption isotherms were carried out on Micromeritics TriStar II 3020 analyzer at $-195.800{ }^{\circ} \mathrm{C}$ under a continuous adsorption condition with equilibration interval of 5 seconds. All samples were pretreated for 12 hour at $200^{\circ} \mathrm{C}$ under nitrogen before measurements. The pore size distribution was calculated from adsorption branches of isotherms by the Barrett-Joyner-Halenda method. Pore volume and specific surface area were calculated by using Barrett-Joyner-Halenda, Brunauer-Emmett-Teller and Langmuir methods, respectively.

\section{Aminofunctionalization of MSN [5, 6, 7,8]}

Aminogroupis attached to the surface MSN using 3aminopropyltriethoxysilane (APTES). $500 \mathrm{mg}$ of mesoporous silica nanoparticle was taken in a three necked round bottom flask placed on rotamantle and purged with nitrogen gas. Nanoparticles were dehydrated by heating at $100^{\circ} \mathrm{C}$ for $6 \mathrm{hrs}$. Mixture of $2 \mathrm{ml} \mathrm{APTES}$ and $50 \mathrm{ml}$ dehydrated ethanol was added to the round bottom flask. Resulting mixture was reflexed at $77^{\circ} \mathrm{C}$ under nitrogen environment for $10 \mathrm{hrs}$.
Nanoparticle suspension was collected by centrifugation at $5500 \mathrm{rpm}$. Sediment was washed three times with water and ethanol to remove impurities and reactant and dried at $50^{\circ} \mathrm{C}$. Amino functionalized mesoporous silica nanoparticles (APT-MSN) are then resupended by ultrasonicationin ultrapure water. The suspension was then freeze dried.

\section{Determination of degree of amino grafting on MSN [9]}

Ninhydrin was applied to quantifythe primary amine groups on the surface of MSN forming the Ruhemann's purple in a weakly acidic solution. First of all APTES solutions of concentration $0.02,0.04,0.06$ and 0.08 millimolar were prepared in ultrapure water. To $1 \mathrm{ml}$ of each solution $3 \%$ ninhydrin reagent was added and heated in a boiling water bath for 15 minutes and cooled for 30 minutes. Then absorbance of each solution was taken at $570 \mathrm{~nm}$ using Jasco V-630 spectrophotometer. 10 mgof APT-MSN was added into $1 \mathrm{ml}$ of ultrapure water and ultrasonicated.The suspension was mixed with $1 \mathrm{ml}$ of $\mathrm{pH} 5$ actetate buffer solution. $1 \mathrm{ml}$ of $3 \%$ ninhydrin reagent was added to the above mixtureand it was heated at $100{ }^{\circ} \mathrm{C}$ for 30 minutes and cooled. The solution was centrifuged at $5000 \mathrm{rpm}$ and absorbance of supernatant was measured at $570 \mathrm{~nm}$ by sing Jasco V-630 spectrophotometer against a blank reference without APTMSN which was prepared simultaneously along with the sample preparation. By using the calibration plot, the corresponding concentration of amine group ( $\mathrm{M}, \mathrm{mol} / \mathrm{L})$ was determined. The molar quantity of amine grafting on the surface of MSNPs was calculated by the following formula:

$$
\begin{aligned}
& \text { Aminegraftingintermsofmol/gofMSN }(A / G)=\frac{M_{A P T E S}}{W_{M S N}} \\
& \text { Aminegraftingintermsof Molecules } / \mathrm{nm}^{2}=\frac{A / G \times 6.022 \times 10^{5}}{S_{B E T}} \\
& \text { AminegraftingtermsofweightpercentageofMSN }=\left(A / G \times W_{A M P}\right) \times 100 \\
& \text { Graftratio }=\frac{A / G \times W_{M S N}}{\text { InitialmolesofAPTESaddedduringfucntionalization }} \times 100
\end{aligned}
$$

Where $\mathrm{M}_{\mathrm{APTES}}$ is the moles of APTES determined from calibration plot, A/G is the moles of APTES grafted on per gram of MSN. $\mathrm{S}_{\mathrm{BET}}$ is the specific surface area of MSN $\left(\mathrm{m}^{2} / \mathrm{g}\right) . \mathrm{W}_{\text {AMP }}$ isthe molecular weight of aminopropylsilylgroup $(86.1861 \mathrm{~g} / \mathrm{mol}) . \mathrm{W}_{\mathrm{MSN}}$ is the weight of MSN added during functionalization.

FTIR scanning of APT-MSN was performed using Agilent technologies CARY 630 FTIR. FTIR spectra were taken at 4 $\mathrm{cm}^{-1}$ resolution, averaged over 32 scans in range of 650 to $4000 \mathrm{~cm}^{-1}$.

Zeta potential was determined by the electrophoretic mobility of APT-MSN in U- type tube at $25^{\circ} \mathrm{C}$, using Zetasizer Nano ZS (Malvern Instruments Ltd, Worcestershire, United Kingdom) after suitable dilution with water.

\section{Drug loading}

The drug loading of 5- fluorouracil was done by backfilling approach. 5-FU and APT-MSN were taken in the ratio 1:1. 2 $\mathrm{mg} / \mathrm{mlsolutionof} 5-\mathrm{FU}$ is prepared with ultrapure water. 40 $\mathrm{mg}$ ofAPT-MSN ultrasonically dispersed in $20 \mathrm{ml}$ of 5-FU solution. The solutions were stirred under room temperature for 24 hour. The solution was then centrifuged at $6000 \mathrm{rpm}$ and $1 \mathrm{ml}$ of supernatant was collected. The amount of 5-FU in the supernatant was found out by UV spectrophotometricalyat 
a wavelength of $266 \mathrm{~nm}$ to determine the unentrapped drug. The prepared nanoparticles were washed and freeze dried. They were subjected to FTIR spectroscopy to confirm drug entrapment. The drug loading capacity and drug entrapment efficiency wascalculated using the following equations.[10]

Drug loading efficiency (\%)

$$
=\frac{\text { Weight of } 5 F U \text { in } A P T-M S N}{\text { weight of } 5 F U \text { loaded } A P T-M S N} \times 100
$$

Drug entrapment efficiency (\%)

$$
=\frac{\text { Weight of } 5 \mathrm{FU} \text { in APT }- \text { MSN }}{\text { Initial weight of } 5 \mathrm{FU}} \times 100
$$

\section{RESULTS AND DISCUSSIONS}

\section{Synthesis ofmesoporoussilica nanoparticles}

The FTIR spectra of MSN was obtained and the spectrumindicates the peaks at $1630 \mathrm{~cm}^{-1}$ (stretching vibration $-\mathrm{OH}$ ), $805 \mathrm{~cm}^{-1}$ (flexible vibration of Si-O) and $1090 \mathrm{~cm}^{-1}$ (stretching vibration Si-O-Si) 3300-3500 $\mathrm{cm}^{-1}$ (Si-O-H stretching vibrations) shown in (Fig1).

The mean zetapotential value obtained is $-27.8 \pm 3.1$ shown in (Fig 2). Zeta potential was uniform distributed. The negative zeta potential value was attributed to the silanolgroups present on the active surface of MSN.

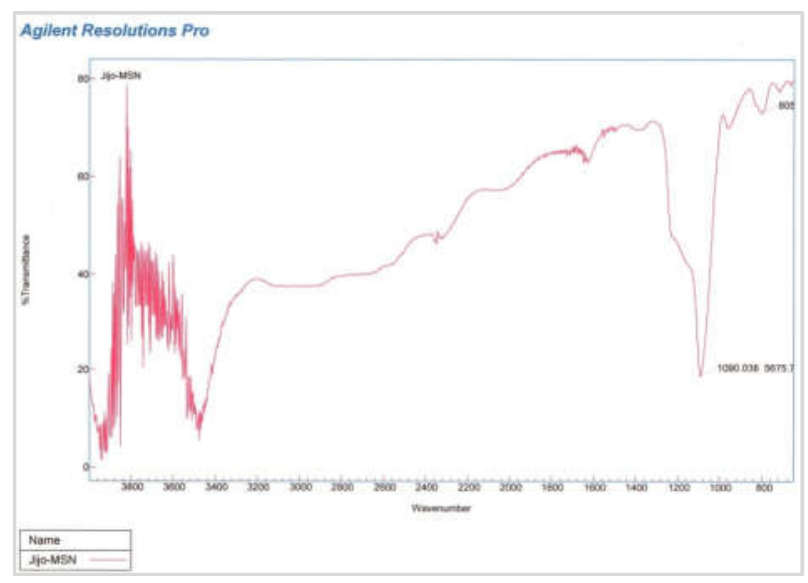

Fig. 1 FTIR spectrum for MSN

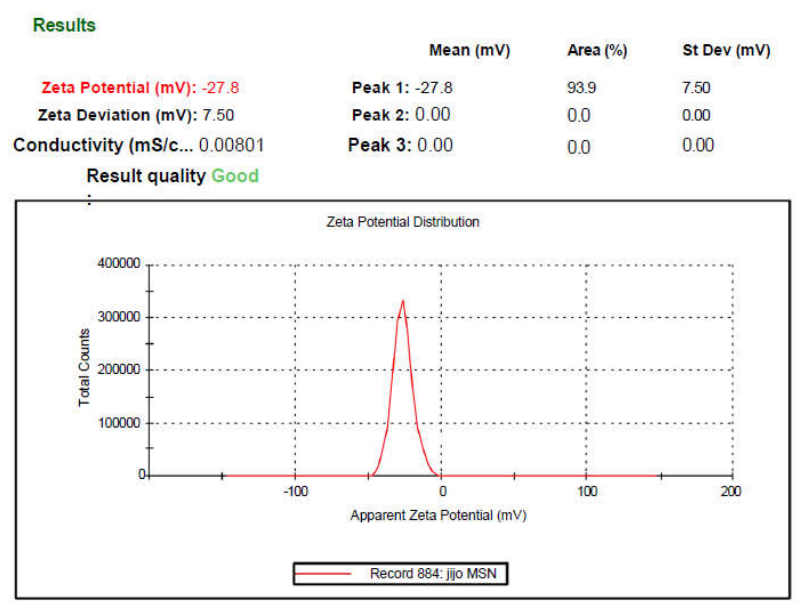

Fig 2 Zeta potential of MSN

The average diameter of the MSN was found to be $154.1 \pm 7.2$ nm shown in (Fig 3). It was also confirmed thatMSN have uniform size distribution.
SEM images of the optimized MSN shown in (Fig 4). From the images it was confirmed that the particles have uniform size distribution and spherical shape.

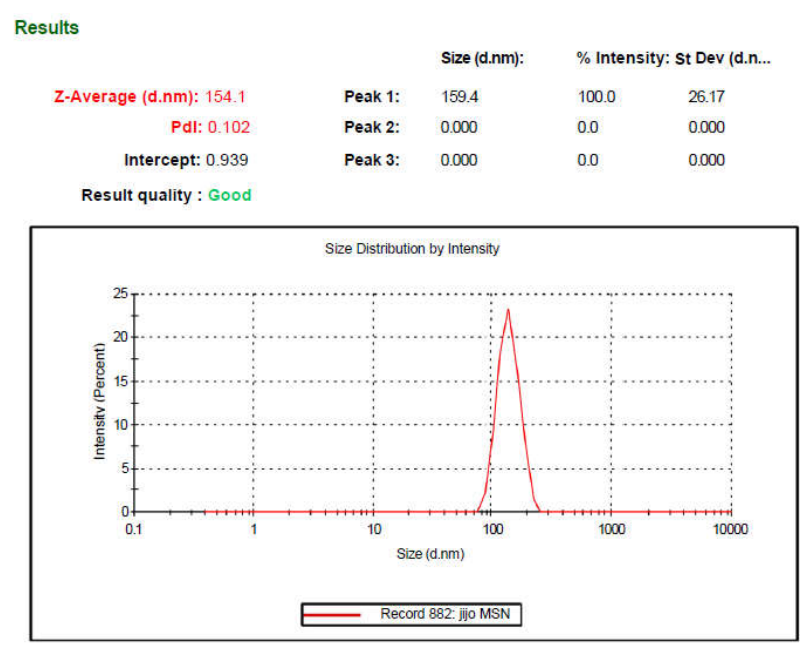

Figure 3 Particle size of MSN

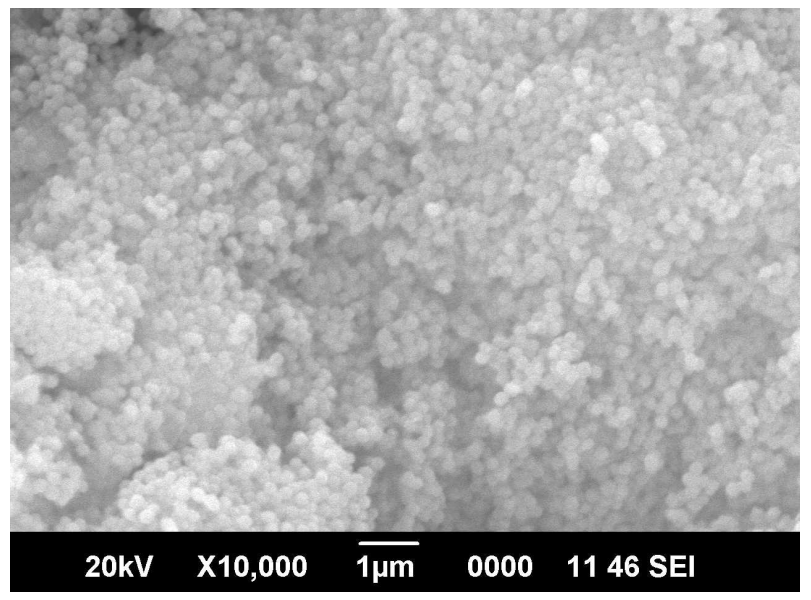

Fig. 4 SEM image of MSN

The TEM images confirm the spherical shape and uniform size distribution of the optimized MSN shown in (Fig 5). The mesoporous structure of MSN was also be confirmed from the image.

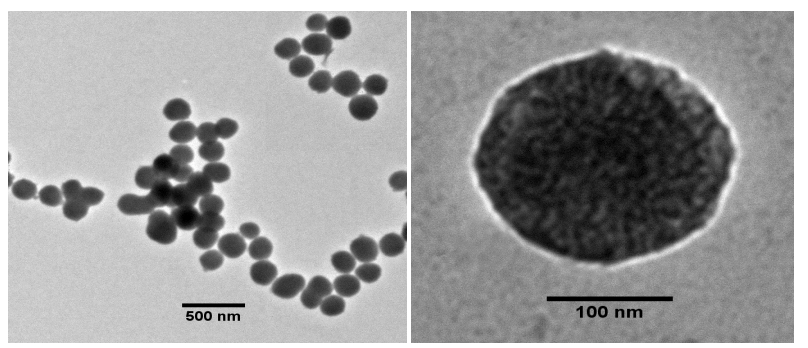

Fig 5 TEM image of MSN

From the surface area analysis it was confirmed MSN was highly porous in nature and it is having a BET surface area of MSN $860.1294 \mathrm{~m}^{2} / \mathrm{g}$. The single point adsorption total pore volume of pores less than $1,060.167 \AA$ diameter at $\mathrm{P} / \mathrm{Po}=$ 0.981148056 was found to $\mathrm{be} 0.708318 \mathrm{~cm}^{3} / \mathrm{g}$. Average pore size of MSN was $2.15 \mathrm{~nm}$ (from adsorption pore distribution).

The amino functionalization of MSN by APTES was depicted in (Fig 6). The amino functionalization was clearly evidenced by the bands detected at around $1500 \mathrm{~cm}^{-1}$ due to 
$\mathrm{N}-\mathrm{H}$ bending and $3000 \mathrm{~cm}-1$ attributed to $\mathrm{C}-\mathrm{N}$ stretching vibrations shown in (Fig 7). The absorption peaks at $2875 \mathrm{~cm}^{-}$ 1 and $2945 \mathrm{~cm}^{-1}$ in the sspectrum were related to $\mathrm{C}-\mathrm{H}$ stretching vibration of aminopropylgroup. The result observed was in close agreement with reported data.

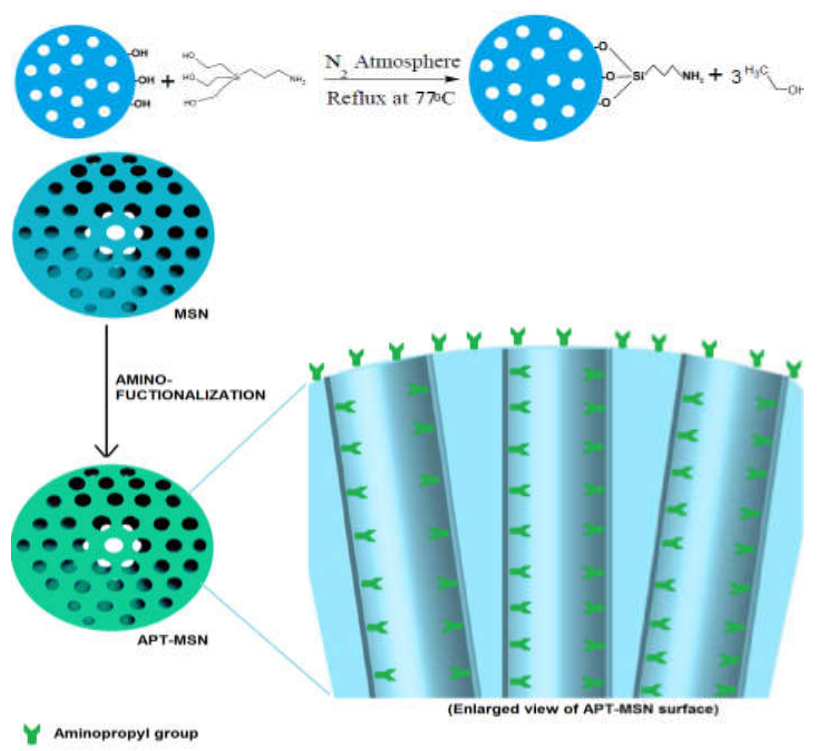

Fig 6 Amino functionalization of MSN

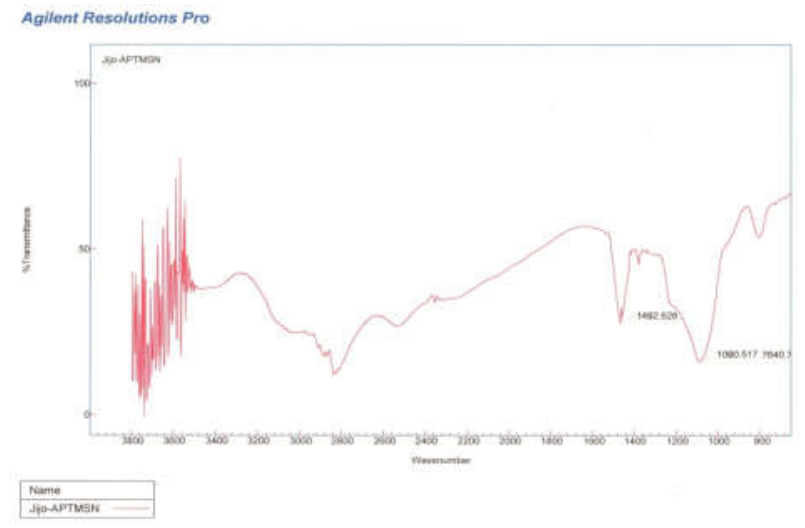

Fig. 7 FTIR spectrum of APT-MSN

Zeta potential of APT-MSN was found to be +6.4 shown in (Fig 8). In contrast to negative zeta potential of the precursor MSN, APT-MSN showed positive charge on the surface. It was attributed to the amino groups attached to the surface during functionalization. The amino groups undergo protonation which makes the surface charge positive.

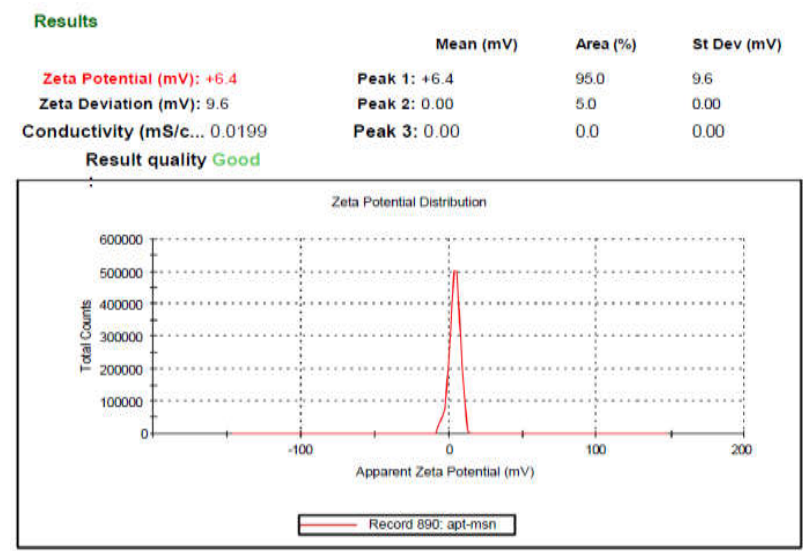

Fig 8 Zeta potential of APT-MSN
The mean particle size of APT-MSN was found to increase after functionalization, due to attachment of aminopropyl group on to the surface during the process shown in (Fig 9).

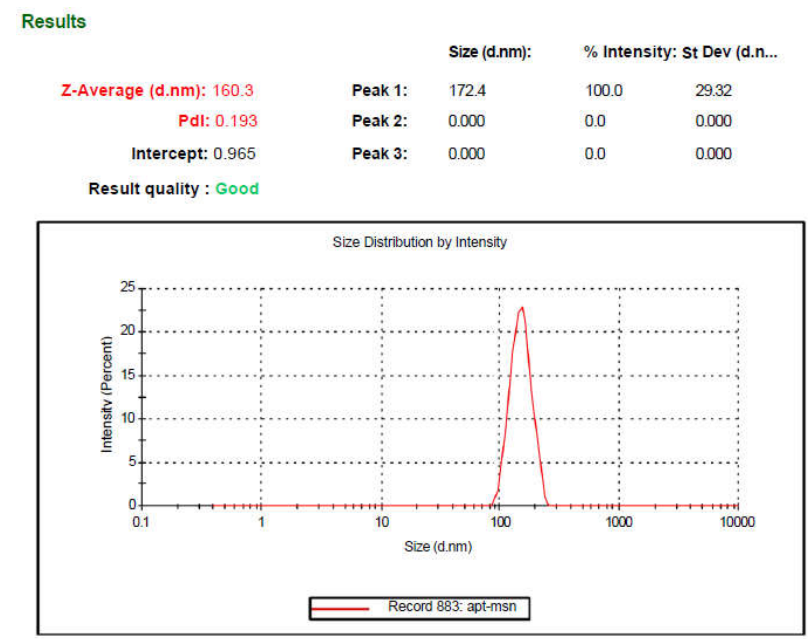

Fig.9 Particle size of APT-MSN

\section{Determination of degree of amino grafting on MSN}

Amine grafting in terms of $\mathrm{mol} / \mathrm{g}$ of $\mathrm{MSN}(\mathrm{A} / \mathrm{G})=$ $0.005841 \pm 0.000178 \mathrm{~mol} / \mathrm{g}$

Amine grafting in terms of Molecules $/ \mathrm{nm}^{2}=4.09=\sim 4$

Amine grafting terms of weight percentage of $\mathrm{MSN}=50.34 \%$ Graft ratio $=30.71 \%$

Zeta potential of APT-MSN was found to be $+6.4 \pm 3.7 \mathrm{mV}$. In contrast to negative zeta potential of the precursor MSN, APT-MSN showed positive charge on the surface. It was attributed to the amino groups attached to the surface during functionalization.

\section{Drug loading}

The process of drug loading was depicted in (Fig 10). The APT-MSN showed significantly greater entrapment efficiency when compared to MSN. The $-\mathrm{NH}_{2}$ groups present on the surface of APT-MSN can significantly increase the amount of 5-FU entrapped into nanoparticles due to the existence of positively charge provided by $-\mathrm{NH}_{2}$ groups. Therefore, the control of the intensity of electrostatic force between nanoparticles and 5-FU may regulate drug loading capacity of MSN. Drug loading to APT-MSN was confirmed by FTIRspectroscopy shown in (Fig 11)

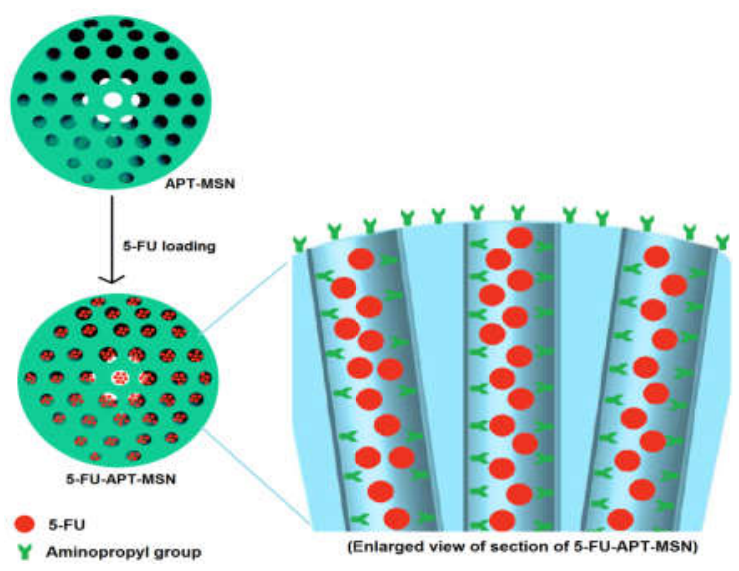

Fig.10 Representation of drug loading to APT-MSN 


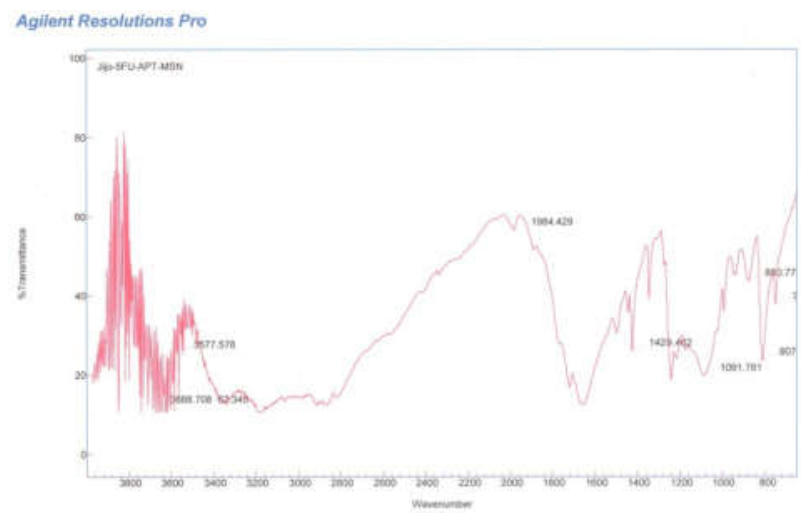

Fig.11 FTIR spectrum of 5-FU-APT-MSN

Entrapment efficiency of $75.47 \%$ was attained at $\mathrm{pH} 8$.

\section{CONCLUSION}

The amino functionalized mesoporous silica nanoparticles were successfully synthesized and was proved for better drug loading of 5-fluorouracil. Its characterization were done and a greater entrapment efficiency of 75.47 was attained at $\mathrm{PH} 8$.

\section{References}

1. Bharti C, Gulati N, Nagaich U, Pal A. Mesoporous silica nanoparticles in target drug delivery system: A review. Int J Pharm Investig. 2015;5(3):124- 133.

2. Tang F, Li L, Chen D. Mesoporous silica nanoparticles: synthesis, biocompatibility and drug delivery. Adv Mater. 2012;24(12):1504-1534.
3. Waldron K, Wu WD, Wu Z, Liu W, Selomulya C, Zhao D, et al. Formation of monodispersemesoporous silica microparticles via spray-drying. $J$ Colloid Interface Sci. 2014; 418:225-233.

4. Cai Q, Luo ZS, Pang WQ, Fan YW, Chen XH, Cui FZ. Dilute solution routes to various controllable morphologies of MCM-41 silica with a basic medium. Chem Mater. 2001; 13(2):258-263.

5. Wang S, Zhang Y, Zhi Z, Jiang T, Zhang J, Wang Z. Spherical mesoporous silica nanoparticles for loading and release of the poorly water-soluble drug telmisartan. J Control Release. 2010; 145(3):257-263.

6. Chang B, Guo J, Liu C, Qian J, Yang W. Surface functionalization of magnetic mesoporous silica nanoparticles for controlled drug release. J Mater Chem. 2010; 20:9941-9947.

7. $\mathrm{Hu}$ L, Sun C, Song A, Chang D, Zheng X, Gao Y, et al. Alginate encapsulated mesoporous silica nanospheres as a sustained drug delivery system for the poorly water-soluble drug indomethacin. Asian $J$ Pharm Sci. 2014; 9(4):183- 190.

8. She X, Chen L, Li C, He C, He L, Kong L. Functionalization of hollow mesoporous silica nanoparticles for improved 5-fu loading. J Nanomater. 2015; Article ID 872035:1-9.

9. Lu H-T. Synthesis and characterization of aminofunctionalized silica nanoparticles. Colloid $J$. 2013;75(3):311-8.

10. Feng W, Nie W, He C, Zhou X, Chen L, Qiu K, et al. Effect of $\mathrm{pH}$-responsive alginate/chitosan multilayers coating on delivery efficiency, cellular uptake and biodistribution of mesoporous silica nanoparticles based nanocarriers. ACS Appl Mater Interfaces. 2014;6(11):8447-8460.

\section{Please cite this article in press as:}

Jijo Thomas., Kala D and Archana George (2017), Aminofunctionalized Mesoporoussilica Nanoparticlesloaded With 5Fluorouracil, International Journal of Current Advanced Research, 6(3), pp. 2394-2398.

http://dx.doi.org/10.24327/ijcar.2017. 2398.0016 